\title{
Management of Astigmatism with the LENSAR Laser System with Streamline
}

\author{
Mark Packer
}

Mark Packer MD Consulting, Inc., Boulder, CO, US

$\mathrm{M}$ anagement of astigmatism at the time of cataract or refractive lens surgery has evolved to include arcuate keratotomy and toric intraocular lens (IOL) implantation. Integration of preoperative corneal diagnostic instrumentation via Streamline digital wireless connectivity to the LENSARTM femtosecond laser (LENSAR Inc., Orlando, FL, US) now allows improved precision and accuracy of arcuate incision and toric IOL alignment, eliminating a significant source of error in astigmatism correction. Use of a pre-programmed, surgeondefined nomogram for arcuate incision construction, based on transmitted data from preoperative corneal analysis, permits further efficiency and reduction of transcription error. Toric IOL alignment is currently facilitated with IntelliAxis ${ }^{T M}$ (LENSAR Inc., Orlando, FL, US) corneal marks. Management of astigmatism at the time of cataract surgery provides the refractive benefit of reduced dependence on glasses and sets the stage for correction of presbyopia

\section{Keywords}

Femtosecond laser, astigmatism, keratotomy, arcuate incision, limbal relaxing incision, toric intraocular lens

Disclosure: Mark Packer is a consultant to LENSAR, Inc. Advanced Vision Science, Inc., Rayner Intraocular Lenses, Ltd., Bausch \& Lomb (Valeant Pharmaceuticals), Medical Monitor, Alcon Research, Ltd., Chief Medical Officer, Equity Holder, i-Optics Corporation (Cassini USA), Chief Medica Officer, Equity Holder, International Biomedical Devices, Inc., STAAR Surgical, Inc., ClearSight, LLC, and Keranova.

Compliance with Ethics: This study involves a review of the literature and did not involve any studies with human or animal subjects performed by any of the authors.

Authorship: All named authors meet the International Committee of Medical Journal Editors (ICMJE) criteria for authorship of this manuscript, take responsibility for the integrity of the work as a whole, and have given final approval to the version to be published.

open Access: This article is published under the Creative Commons Attribution Noncommercial License, which permits any noncommercial use, distribution, adaptation, and reproduction provided the original author(s) and source are given appropriate credit.

Received: August 9, 2017

Accepted: October 6, 2017

Citation: US Ophthalmic Review, 2017;10(2):99-103

Corresponding Author: Mark Packer, Mark Packer MD Consulting, Inc., 1400 Bluebell Ave, Boulder, CO 80302. E: mark@markpackerconsulting.com

Support: The publication of this article was supported by LENSAR., Inc.
Through the centuries, ceaseless innovation has advanced cataract surgery - the most frequently performed operation on earth - from Daviel's lens extraction, to Ridley's intraocular lens (IOL) implantation, to Kelman's phacoemulsification, and now to femtosecond laser assisted techniques.' Femtosecond laser surgery represents the marriage of two technologies: ocular imaging and laser photolysis. Most lasers depend on intraoperative optical coherence tomography imaging. The LENSAR ${ }^{\text {TM }}$ femtosecond laser (LENSAR Inc., Orlando, FI, US) utilizes proprietary augmented reality (AR) imaging and anterior segment biometry based on scanning structured illumination. Super luminescent diode technology provides the illumination for AR and scans at a variable rate depending on the target structure, ensuring optimal contrast for structures with higher light scatter, such as the cornea, as well as for those with little scatter, such as the posterior lens capsule. The 3D-AR software locates anatomic interfaces including the pupil, anterior and posterior corneal surfaces and the anterior and posterior lens capsule. The multiple images are collated using optical ray tracing techniques to generate an exact 3D reconstructed model of the anterior segment.

In the LENSAR system, intraoperative imaging through structured illumination allows reconstruction of a 3D model of the anterior segment of the eye, which is used to guide laser incisions. ${ }^{2}$ In addition to intraoperative imaging, wireless digital communication technology now permits integration of preoperative imaging into laser guidance. Recent US Food and Drug Administration (FDA) 510(K) clearances have demonstrated the utility of linkage between preoperative diagnostic instruments and the LENSAR laser, including the Cassini Corneal Shape Analyzer (Cassini, The Hague, The Netherlands), the Corneal Analyzer OPD-Scan III (Nidek, Aichi, Japan), the Aladdin (Topcon Corporation, Tokyo, Japan) and both the Pentacam ${ }^{\circledR}$ HR and the Pentacam ${ }^{\circledR}$ AXL (Oculus, Wetzlar, Germany). The key to these linkages is iris registration, the accurate mapping and matching of iris features from the preoperative image captured in the clinic to the intraoperative image captured through the laser optics. Iris registration, based on high definition preoperative infrared images obtained with these corneal diagnostic instruments, allows precise correlation of corneal topographic and total corneal astigmatic data with laser treatment, opening the way for accurate correction of corneal astigmatism.

Correction of corneal astigmatism represents the entry point to refractive cataract surgery because it is prerequisite to achieving spectacle independence for the majority of surgical candidates. The introduction of toric multifocal and toric extended depth of focus IOL optical designs has raised the bar for correction of astigmatism and presbyopia at the time of cataract surgery; however, demonstration of the effectiveness of toric IOLs for correction of lower levels of corneal astigmatism, particularly $<1.00 \mathrm{D}$, has remained elusive. For example, approved labeling for the Tecnis ${ }^{\circledast}$ Toric IOL 
(Johnson \& Johnson Vision Care, Santa Ana, CA, US) states, "Study results (residual refractive cylinder, change in cylinder, and uncorrected acuity) stratified by preoperative keratometric cylinder did not show evidence of significant benefit in the treatment of preoperative corneal astigmatism of less than one diopter. ${ }^{\text {"3 }}$ Because uncorrected visual acuity can nevertheless be compromised by mild astigmatism from 0.25 to $1.25 \mathrm{D}$, and because approximately two thirds of candidates for cataract surgery have preexisting corneal astigmatism in this range, ${ }^{4}$ corneal relaxing incisions and arcuate keratotomies performed at the time of cataract surgery have remained popular. While manual limbal relaxing incisions and arcuate keratotomies have allowed reduction of mild to moderate pseudophakic astigmatism, ${ }^{5}$ the advent of femtosecond laser arcuate incisions has increased automation and standardization of treatment parameters. However, few reports of the effectiveness of femtosecond laser arcuate incisions in the context of cataract surgery have appeared in the literature.

Even when utilizing the femtosecond laser, manual marking of the corneal steep axis can introduce significant sources of error in the correction of astigmatism. The same issues may arise, of course, with corneal marking in preparation for alignment of toric IOLs. For example, in their recent study of femtosecond laser arcuate incisions for the correction of astigmatism at the time of cataract surgery, chan et al. marked a single reference point at the temporal limbus with the patient sitting upright to compensate for cyclorotation. Once the patient was transferred to the femtosecond laser platform, the temporal limbus was used as a reference point for the zero-degree mark on a ring gauge. Nevertheless, these authors admit "treatment misalignment was a major influential error-inducing factor in astigmatism correction." ${ }^{\prime 7}$

Accurate alignment of toric IOLs, and arcuate incisions, represents a critical step in the correction of pre-existing corneal astigmatism. Not only has it been estimated that there is 3.3\% loss of effect for every degree of offaxis correction, ${ }^{8}$ but it has also been suggested off-axis correction creates a new vector resulting in abnormal induced astigmatism on an entirely different axis, and induces higher order aberrations. ${ }^{9}$

Utilization of iris registration coupled with image-guided femtosecond laser incision construction has the potential to improve accuracy and provide superior refractive outcomes by overcoming the drawbacks of manual marking techniques. LENSAR has developed Streamline technology with this goal in mind, to permit accurate and reproducible reduction or elimination of mild to moderate astigmatism in conjunction with femtosecond laser assisted cataract surgery.

\section{LENSAR with Streamline system}

The acquisition of preoperative corneal topography, or total corneal astigmatic analysis including the posterior corneal surface, with high definition infrared digital images of iris features, forms the basis of imageguided laser arcuate incision placement and construction. The corneal diagnostic instrument can verify image compatibility at the point of capture to minimize the risk of failure of cyclorotation compensation due to the discovery of inadequate imaging once the patient is already in the operating room. Streamline wirelessly transfers preoperative data from one of the linked instruments (Cassini Corneal Shape Analyzer, OPD, Aladdin, Pentacam HR or Pentacam AXL) to the LENSAR laser, reducing the number of steps within the patient workflow and eliminating the need for staff to transfer the correct data either manually or via a memory device. * At the same time, wireless automated data transmission into the LENSAR patient planning software eliminates potential transcription errors.

Iris registration is accomplished through selection and matching of iris features from the preoperative, undilated pupil to the intraoperative, dilated pupil. ${ }^{10}$ Streamline automatically corrects for cyclorotation after the patient is docked to the laser, and does not necessitate that the surgeon visually inspect and verify that cyclorotation compensation was accurate. Streamline iris registration cannot be affected by loss of vascular detail due to pharmacologic effects.

Arcuate incision planning software auto-populates incision parameters based on the surgeon's preferred nomogram and pre-programmed surgically induced astigmatism (SIA). Arcuate incision planning can automatically recommend laser incision placement based on preoperative data; alternatively, there is the capability for manual entry or adjustment of preoperative data. In addition, arcuate incision planning provides a graphical interface to demonstrate SIA and calculated residual astigmatism. This nomogram-based planning tool optimizes arcuate incision construction by allowing modifications based on patient age and up to three additional parameters, such as corneal white-to-white, central corneal thickness, and corneal slope rate-of-change.

Multiple metrics can be customized to optimize arcuate incision planning; these include incision depth, with options for fixed depth (250-900 $\mu$ ), fixed residual depth (0-300 $\mu$ ), and percentage thickness (10-100\%). 'Against the Rule' and 'With the Rule' surgeon tables allow for entry of multiple data points for arcuate incision length based on astigmatic power preferences. Multiple data points can be added to better define arcuate incision planning, such as single- or paired-arc treatments, modifiers, or surgeon preferences. In addition, an age modifier provides the surgeon with the option to adjust arcuate length utilizing an age-based chart. Finally, the incision placement pop-up box allows the surgeon to adjust clear corneal incision (CCl) locations preoperatively utilizing surgeon tables and SIA information in order to minimize residual postoperative astigmatism.

Surgeons adopting LENSAR with Streamline arcuate incisions must develop treatment nomograms and criteria for candidate selection. While femtosecond laser arcuate incision nomograms have appeared in the literature, nomogram development and refinement require tracking and analyzing clinical outcomes. ${ }^{11}$ LENSAR with Streamline also has demonstrated utility in the alignment of toric IOLs through the IntelliAxis ${ }^{\mathrm{TM}}$ corneal marking system (LENSAR Inc., Orlando, FI, US), which produces visible steep axis landmarks on the cornea that may be used by the surgeon to verify the location of the steep axis relative to toric IOL orientation.

\section{Clinical Results with LENSAR arcuate incisions}

We have conducted a search of the published literature using PubMed. gov, as well as relevant scientific meeting abstracts, using the search terms "LENSAR" and "astigmatism," in order to elucidate the safety and effectiveness of LENSAR Streamline arcuate incisions. Several authors have reported clinical outcomes with LENSAR with Streamline arcuate incisions. At the American Society of Cataract and Refractive Surgery 2016 Annual Meeting, in New Orleans, Mitchell Jackson, MD presented data on 52 eyes of 31 patients with pre-existing astigmatism ranging between 0.4 to $2.75 \mathrm{D} .^{12}$ All patients underwent LENSAR femtosecond laser assisted arcuate incisions during cataract surgery. A significant reduction in the mean 
Figure 1: Mean absolute value of refractive astigmatism following LENSAR femtosecond laser assisted arcuate incisions during cataract surgery

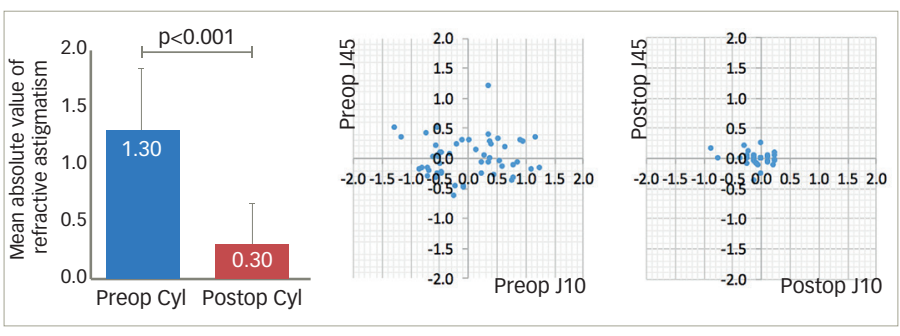

Figure 2: Residual refractive astigmatism

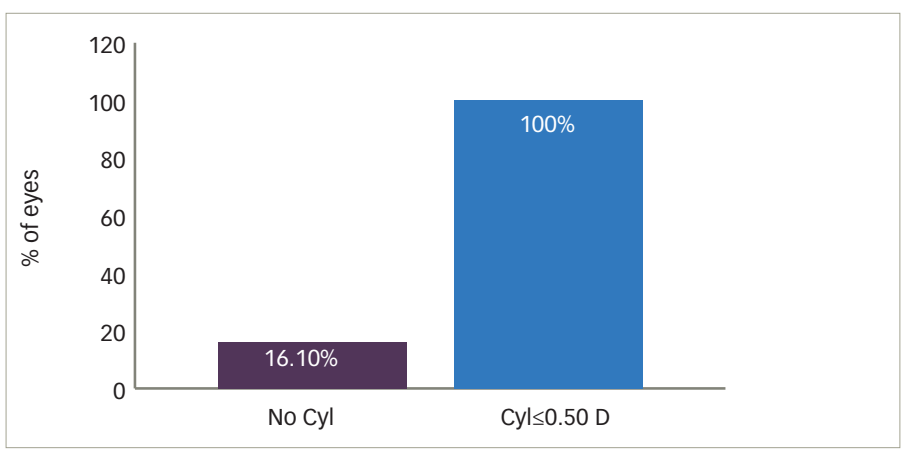

absolute value of refractive astigmatism from $1.3 \mathrm{D}$ to $0.3 \mathrm{D}(\mathrm{p}<0.001)$ was observed. Correspondingly, vector analysis of refractive cylinder also revealed an improvement in the J0 and J45 vectors. No complications were observed (Figure 1). The author concluded that LENSAR laser assisted astigmatism correction by arcuate incision construction during cataract surgery, is a safe and effective treatment option.

Also at the at the American Society of Cataract and Refractive Surgery 2016 Annual Meeting in New Orleans, Jonathan Solomon, MD presented data on 31 eyes with regular corneal astigmatism treated with the LENSAR laser with Streamline, to perform partial thickness arcuate incisions with implantation of a monofocal IOL.13 Postoperatively, $90.3 \%$ of eyes were within $1.00 \mathrm{D}$ and $83.9 \%$ of eyes were within $0.50 \mathrm{D}$ of the spherical equivalent target refraction. 100\% of eyes achieved postoperative refractive astigmatism $\leq 0.50 \mathrm{D}$ (Figure 2). In addition, corneal simulated keratometric astigmatism as measured by the Cassini Corneal Shape Analyzer was reduced significantly from a preoperative mean of $1.25 \mathrm{D}$ to $0.40 \mathrm{D}$ postoperatively (Figure 3).

The author concluded that precise and reproducible arcuate incisions can be constructed with the LENSAR laser.

At the American Society of Cataract and Refractive Surgery 2017 Annual Meeting in Los Angeles, Denise Visco, MD presented a retrospective study comprising data from 279 eyes of 203 patients with cataract and pre-existing keratometric astigmatism ranging from $0.50 \mathrm{D}$ to 1.91 D. ${ }^{14}$ All eyes underwent LENSAR femtosecond laser assisted cataract surgery and arcuate incision construction using Streamline wireless transfer of the preoperative undilated iris registration image and corneal astigmatism data from the Cassini Corneal Shape Analyzer. Cyclorotation
Figure 3: Cassini keratometric simulated K (D)

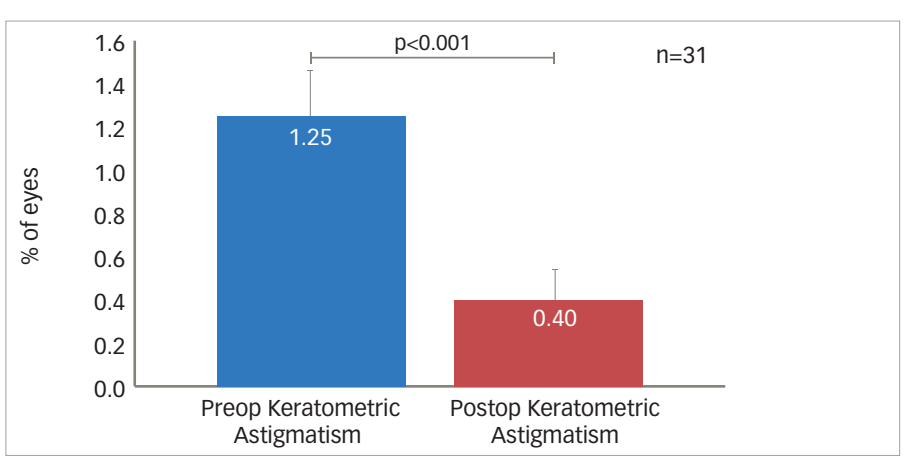

was automatically compensated by adjusting incision placement, and the incision parameters were automatically generated by the LENSAR arcuate incision planning software based on the surgeon entered nomogram and personalized surgically induced astigmatism.

The primary outcome measure was postoperative residual refractive astigmatism. Preoperatively, mean keratometric astigmatism measured $0.92 \pm 0.33 \mathrm{D}$; postoperatively, mean refractive astigmatism measured 0.09 $\pm 0.21 \mathrm{D}$ (Figure 4). $94.6 \%$ eyes had $\leq 0.5 \mathrm{D}$ and $99.3 \%$ eyes had $\leq 0.75 \mathrm{D}$ residual refractive astigmatism (Figure 5). A double angle plot graphically demonstrates significant reduction of preoperative keratometric astigmatism to postoperative refractive astigmatism 3 months after surgery (Figure 6). There were no intraoperative complications. The author concluded that LENSAR with Streamline arcuate incision planning software using iris registration yielded safe and effective outcomes in cataract patients with low to moderate astigmatism. ${ }^{14}$

\section{Discussion-LENSAR arcuate incision clinical outcomes}

These results, demonstrating $94.6-100 \%$ of eyes $\leq 0.50 \mathrm{D}$ residual refractive astigmatism, compare favorably with published outcomes of femtosecond laser arcuate incisions performed at the time of cataract surgery, utilizing other laser platforms. In their study of 51 eyes of 37 patients operated with the LenS $x^{\otimes}$ laser (Alcon Laboratories, Inc., Fort Worth, TX, US), with mean preoperative keratometric astigmatism of 1.45 $\pm 0.44 \mathrm{D}$ (range, $0.50-2.50$ ), wang et al. reported $50 \%$ of eyes $\leq 0.50 \mathrm{D}$ residual refractive astigmatism at 3 months postoperative. ${ }^{15}$ Day et al. reported $32.1 \% \leq 0.50 \mathrm{D}$ residual refractive astigmatism in a cohort of 196 eyes of 133 patients, with mean preoperative keratometric astigmatism of $1.21 \pm 0.42 \mathrm{D}$ (range, $0.75-2.64$ ) operated with the Catalys ${ }^{\oplus}$ laser system (Johnson \& Johnson Vision Care, Santa Ana, CA, US). ${ }^{6}$ In their study of 54 eyes of 54 patients with mean preoperative keratometric astigmatism of $1.33 \pm 0.57 \mathrm{D}$ ( $95 \%$ confidence interval [CI], 1.31-1.35), Chan et al. performed arcuate incisions using the VICTUS ${ }^{\circledR}$ laser (Bausch \& Lomb, Dornach, Germany) and reported 33\% of eyes $\leq 0.50 \mathrm{D}$ residual refractive astigmatism. ${ }^{16}$ The higher means and upper limits of preoperative keratometric astigmatism of eyes enrolled in these studies may have impacted postoperative residual refractive astigmatism, and the outcomes suggest that eyes with $>2.00 \mathrm{D}$ pre-existing corneal astigmatism are likely better candidates for toric IOL implantation. Visco's study highlights the most effective range of femtosecond laser arcuate incisions, from $0.50 \mathrm{D}$ to $2.00 \mathrm{D}$ preoperative keratometric astigmatism. ${ }^{14}$ In addition, utilization of iris registration for accurate placement of 
Figure 4: Postoperative residual refractive astigmatism

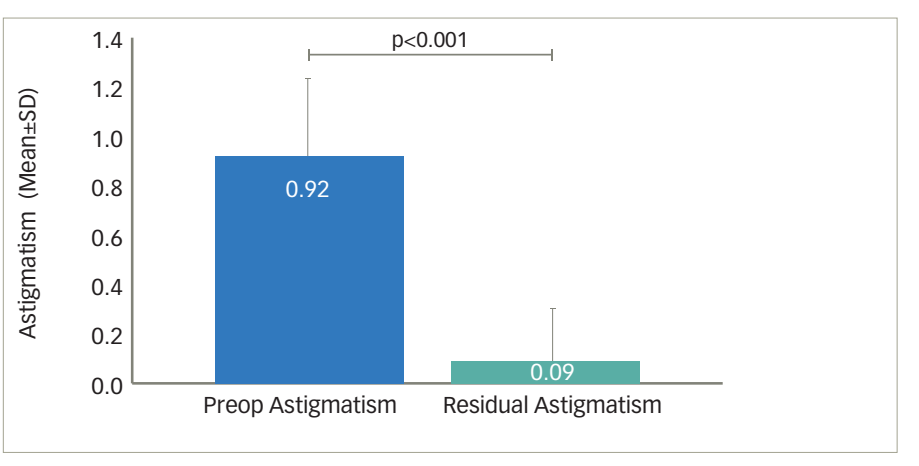

incisions likely reduces error from misalignment. As Chan et al. point out, "The variability in treatment alignment can be the result of inconsistency in aligning the steepest meridian to the incisions." ${ }^{16}$

\section{Clinical results with LENSAR IntelliAxis toric IOL alignment}

For those patients with moderate or greater astigmatism not likely to be completely corrected by arcuate incisions alone, toric IOLs offer a proven refractive option. As mentioned above, LENSAR with Streamline includes the capability of marking the corneal steep axis with IntelliAxis based on wireless transmission of preoperative diagnostic data and iris registration in order to provide landmarks for toric IOL alignment.

Visco and Weinstock presented results of a multicenter, prospective, non-masked clinical study including subjects having astigmatism with cataracts desiring lens extraction and toric $\mathrm{IOL}$ implantation. ${ }^{17}$ Other inclusion criteria were keratometric cylinder between 0.75 D and 4.50 D and successful iris registration for cyclorotation compensation. All subjects in the study underwent LENSAR laser assisted cataract surgery, with the creation of steep axis corneal landmarks (using intrastromal corneal incisions), followed by toric IOL implantation. Toric IOLs were aligned rotationally using the femtosecond steep axis landmark. The subjects were followed at 1 day and 1 month, postoperatively. Seventythree eyes were enrolled in the study with a mean preoperative corneal cylinder of $2.23 \pm 0.19 \mathrm{D}$ (range, 1.15-4.5 D). Postoperatively, at one month, 93.2\% of eyes achieved $\leq 0.50 \mathrm{D}$ and $76.7 \%$ of eyes achieved $\leq 0.25 \mathrm{D}$ residual refractive astigmatism. The mean residual refractive astigmatism was $0.19 \pm 0.38 \mathrm{D}$. These results compare favourably with other studies of toric IOL implantation. ${ }^{18}$

Stephenson presented results of a prospective comparative study in which axis marks based on Cassini Total Corneal Astigmatism (TCA; Cassini, The Hague, The Netherlands), which captures both anterior and posterior corneal cylinder, were placed with the LENSAR femtosecond laser IntelliAxis system. ${ }^{19}$ Intraoperative aphakic measurements were obtained using the ORA $^{\text {TM }}$ intraoperative aberrometer (Alcon, Ft. Worth, TX). Vector analysis was used to calculate the difference between preoperative total corneal astigmatism, femtosecond-guided corneal marks and intraoperative aberrometry-measured astigmatism. Results included data on 74 eyes of 47 subjects undergoing toric IOL implantation. Measurements of corneal astigmatism preoperatively by Cassini TCA and intraoperatively by ORA were highly correlated: Pearson correlation coefficients $(R)$ for axis and magnitude of corneal astigmatism were $0.94 \mathrm{D}$ and $0.72 \mathrm{D}$, respectively
Figure 5: Cumulative percentage distribution of residual astigmatism

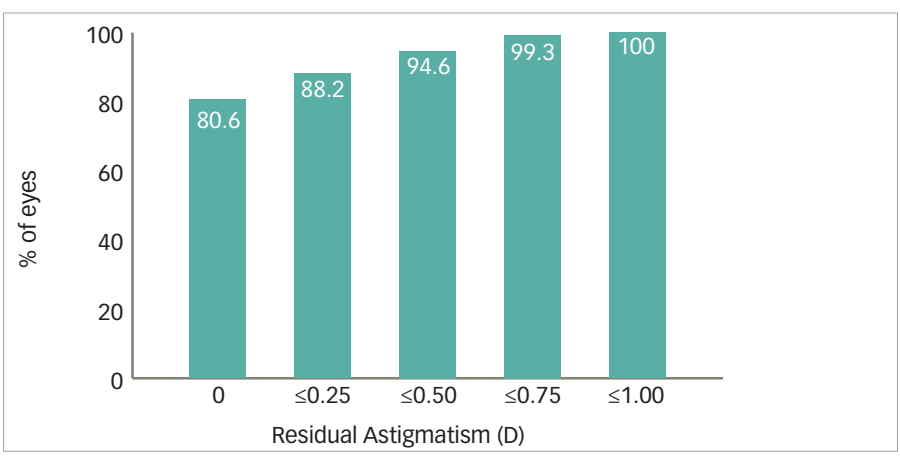

Figure 6: Preoperative keratometric astigmatism to postoperative refractive astigmatism 3 months after surgery

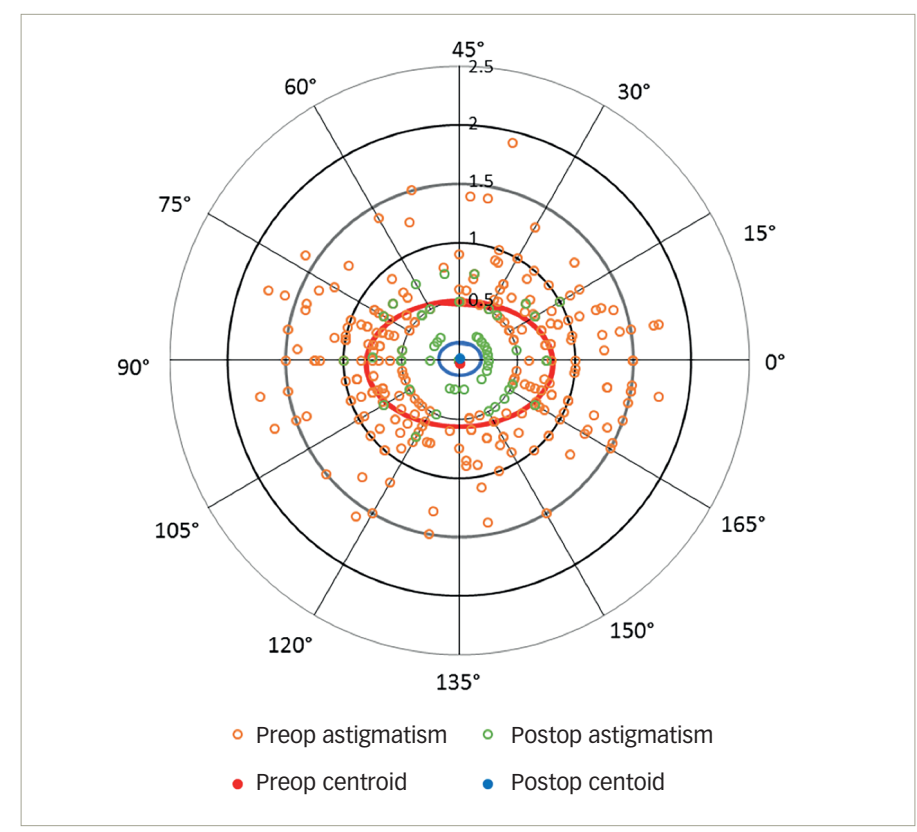

$(p<0.0001$ for each). The author concluded that the correlation between preoperative and intraoperative measurements provided a high level of confidence in selection and alignment of toric IOLS.

\section{Approach to the patient}

Correction of astigmatism is fundamental to refractive cataract surgery. Whether utilizing arcuate incisions for mild astigmatism or toric IOLS for moderate and higher astigmatism, accurate alignment represents a critical step in achieving the full intended correction. Image guided laser incisions and corneal marks for toric IOL positioning, based on preoperative corneal analysis and iris registration, provide highly precise guidance for alignment of astigmatic correction. Nomogram development and adjustment based on postoperative outcomes allow for continuous improvement.

Informed consent at the time of cataract surgery requires discussion of alternative procedures, including refractive correction of astigmatism. Reduction or elimination of spectacle dependence has become a widely 
understood option for cataract patients. Multiple methodologies are offered in practice today, ranging from monovision with arcuate incisions to bilateral toric extended depth of focus IOLs. Corneal refractive procedures continue to find utilization for enhancement of residual refractive error. ${ }^{20}$ Given the complexity of the available options, clear and concise communication with patients has become an essential component of refractive cataract surgery.

From the patient's perspective, there are three options for needing glasses in order to see things clearly: none, single vision glasses, or (usually progressive) bifocals. Image quality can be difficult to explain, but it is most often discussed in terms of the "halos around lights" that accompany multifocal or extended depth of focus IOLs. Through interaction with the patient, the surgeon must determine the patient's degree of motivation for spectacle independence and relative tolerance for unwanted optical side effects, i.e., dysphotopsia and reduced contrast sensitivity. From this assessment should spring a recommendation for the most likely successful technological approach, which should then be explained in as simple a way as possible. Regardless of the decision, clinical results to date show that there may be significant benefit to offering correction of mild astigmatism via femtosecond laser arcuate incisions. The results obtained, and the potential for further improvement through ongoing nomogram adjustment, make this modality an extremely promising route towards spectacle independence. $\square$
1. Packer M, Lindstrom RL, Davis EA, Evolution of Cataract Surgery. In Krueger RR, Talamo JH and Lindstrom RL (Eds) Textbook of Refractive Laser Assisted Cataract Surgery (ReLACS). Springer: New York, 2013; 1-8.

2. Packer M, Klyce SD, Smith C, The LENSAR ${ }^{\circledR}$ Laser System-fs 3D for femtosecond cataract surgery, European Ophthalmic Review 2014;8:93-8.

3. Toric Monofocal One-Piece Posterior Chamber Intraocular Lens (IOL). TECNIS ${ }^{\circledast}$ Toric 1-Piece Intraocular Lens, Models ZCT150, ZCT225, ZCT300, ZCT400 and TECNIS ${ }^{\oplus}$ Toric Calculator System PMA P980040/S039: FDA Summary of Safety and Effectiveness Data, Page 27. Available at: www.accessdata.fda.gov/cdrh_docs/ pdf/P980040S039b.pdf (Accessed July 6, 2017).

4. Ferrer-Blasco T, Montés-Micó R, Peixoto-de-Matos SC, et al. Prevalence of corneal astigmatism before cataract surgery, J Cataract Refract Surg, 2009;35:70-5.

5. Packer $M$, Effect of intraoperative aberrometry on the rate of postoperative enhancement: retrospective study, J Cataract Refract Surg, 2010;36:747-55.

6. Day AC, Lau NM, Stevens JD, Nonpenetrating femtosecond lase intrastromal astigmatic keratotomy in eyes having cataract surgery, J Cataract Refract Surg, 2016;42:102-9.

7. Chan TC, Ng AL, Cheng GP, et al., Corneal astigmatism and aberrations after combined femtosecond-assisted phacoemulsification and arcuate keratotomy: two-year results,
Am J Ophthalmol, 2016;170:83-90

8. Novis C, Astigmatism and toric intraocular lenses, Curr Opin Ophthalmol, 2000;11:47-50

9. Motwani $\mathrm{M}$, The use of WaveLight ${ }^{\boxplus}$ Contoura to create a uniform cornea: the LYRA Protocol. Part 2: the consequences of treating astigmatism on an incorrect axis via excimer laser, Clin Ophthalmol, 2017:11:907-13.

10. Chernyak $D A$, Iris-based cyclotorsional image alignment method for wavefront registration, IEEE Trans Biomed Eng, 2005;52:2032-40

11. Blehm C, Potvin R, Pseudophakic astigmatism reduction with femtosecond laser-assisted corneal arcuate incisions: a pilot study, Clin Ophthalmol, 2017;11:201-7.

12. Jackson MA, Treatment of Corneal Astigmatism Using Femtosecond Laser-Assisted Arcuate Incisions During Cataract Surgery. Paper presented at the American Society of Cataract and Refractive Surgery 2016 Annual Meeting. New Orleans, LA. May 7-10, 2016

13. Solomon JD, Efficacy of Femtosecond Laser-assisted Partial Thickness Arcuate Incisions to Reduce Astigmatism. Presented at the American Society of Cataract and Refractive Surgery 2016 Annual Meeting. New Orleans, LA. May 7-10, 2016.

14. Visco DM, Managing Corneal Astigmatism Using Iris Registration Guided, Femtosecond Laser Assisted Arcuate Incisions Performed with Cataract Surgery. Presented at the American Society of
Cataract and Refractive Surgery 2017 Annual Meeting. Los Angeles, CA. May 6-9, 2017

15. Wang L, Zhang $S$, Zhang Z, et al., Femtosecond laser penetrating corneal relaxing incisions combined with cataract surgery, I Cataract Refract Surg, 2016;42:995-1002.

16. Chan TC, Cheng GP, Wang Z, et al., Vector analysis of corneal astigmatism after combined femtosecond-assisted phacoemulsification and arcuate keratotomy, Am J Ophthalmol 2015;160:250-5.e2.

17. Visco DM, Weinstock RJ, Astigmatism Management Using Steep Axis Corneal Landmarks Created with a Femtosecond Laser: Multicenter Prospective Clinical Study. Presented at the American Society of Cataract and Refractive Surgery 2016 Annual Meeting. New Orleans, LA. May 7-10, 2016.

18. Solomon JD, Ladas J, Toric outcomes: Computer-assisted registration versus intraoperative aberrometry, J Cataract Refract Surg, 2017;43:498-504

19. Stephenson PDG. Toric IOL Axis Alignment by Total Corneal Astigmatism with Femtosecond Laser Marking and Intraoperative Aberrometry. Presented at the American Society of Cataract and Refractive Surgery 2017 Annual Meeting. Los Angeles, CA, May 6-9, 2017.

20. Packer M, Enhancements After Premium IOL Cataract Surgery: Tips, Tricks, and Outcomes; in Starr C (ed.), Curr Ophthalmol Rep. New York: Springer Science + Business Media, 2013. 